\title{
Micromagnetic simulation studies of ferromagnetic part spheres
}

\author{
Richard P. Boardman, Jürgen Zimmermann, and Hans Fangohr ${ }^{\mathrm{a})}$ \\ Computational Engineering and Design Group, School of Engineering Sciences, University of Southampton, \\ Southampton SO17 1BJ, United Kingdom
}

\author{
Alexander A. Zhukov and Peter A. J. de Groot \\ School of Physics and Astronomy, University of Southampton, Southampton SO17 1BJ, United Kingdom
}

(Presented on 10 November 2004; published online 6 May 2005)

\begin{abstract}
Self-assembly techniques can be used to produce periodic arrays of magnetic nanostructures. We have developed a double-template technique using electrochemical deposition. This method produces arrays of dots which are of spherical shape, as opposed to those prepared by standard lithographic techniques, which are usually cylindrical. By varying the amount of material that is deposited electrochemically, spheres of diameter $d$ can be grown up to varying heights $h<d$. Thus different spherical shapes can be created ranging from shallow dots to almost complete spheres. Using micromagnetic modeling, we calculate numerically the magnetization reversal of the soft part spherical particles. The observed reversal mechanisms range from single domain reversal at small radii to vortex movement in shallow systems at larger radii and vortex core reversal, as observed in spheres at larger heights. We present a phase diagram of the reversal behavior as a function of radius and growth height. Additionally, we compare simulation results of hybrid finite element/boundary element and finite difference calculations for the same systems. (C) 2005 American Institute of Physics. [DOI: 10.1063/1.1850073]
\end{abstract}

\section{INTRODUCTION}

Magnetic recording media created with traditional sputtering methods nears the fundamental limits as areal bit density increases. Magnetic grains of size orders beneath the paramagnetic limit are unstable at room temperature, making them an ineffective choice for long-term storage media as the many irregular grains used to create a storage bit can no longer be relied on to maintain a particular overall direction of magnetization.

A solution to this problem is to form regular patterned media where data can be stored as one bit per object; the objects in these patterned media can subsequently have a larger size than the grain size in nonpatterned media while increasing the areal density. Photolithographic methods can be used to create these media but are limited to $0.1-\mu \mathrm{m}$ sizes. Techniques which use electron-beam lithography are not cost effective on a large scale. Self-assembly methods, however, seem to be an economic way to create nanostructured magnets. 1

Using a chemical self-assembly technique ${ }^{2}$ templates can be produced through the evaporation of the liquid from an aqueous suspension of latex spheres of diameter $d$, leading to the formation of a close-packed array. Filling the gaps between the spheres with a nonmagnetic material and etching away the latex spheres leave a honeycomblike template which can then be filled with a magnetic material up to a height $h$. Altering the level $h$ to which the honeycomb template is filled allows part-spherical geometries to be produced that range from very shallow, for example, height $h$ $=1 / 8 d$, to almost completely full, as $h=7 / 8 d$.

At the nanometer length scale the magnetic behavior is

${ }^{a)}$ Electronic mail: fangohr@soton.ac.uk strongly influenced by the shape of the structure. Related work has been performed on flat cylinders and discs, ${ }^{3-6}$ spheres, ${ }^{7-9}$ triangles, ${ }^{10}$ and other polygons. ${ }^{10,11}$ In this paper, we study the behavior of the new class of part-spherical structures and compute a magnetic reversal phase diagram as a function of diameter and height.

We further compare the simulation results obtained with the finite difference method ${ }^{12}$ and the hybrid finite element/ boundary element method. ${ }^{13}$

\section{METHOD}

We use the hybrid finite element/boundary element micromagnetic simulation program MAGPAR (Ref. 13) and the

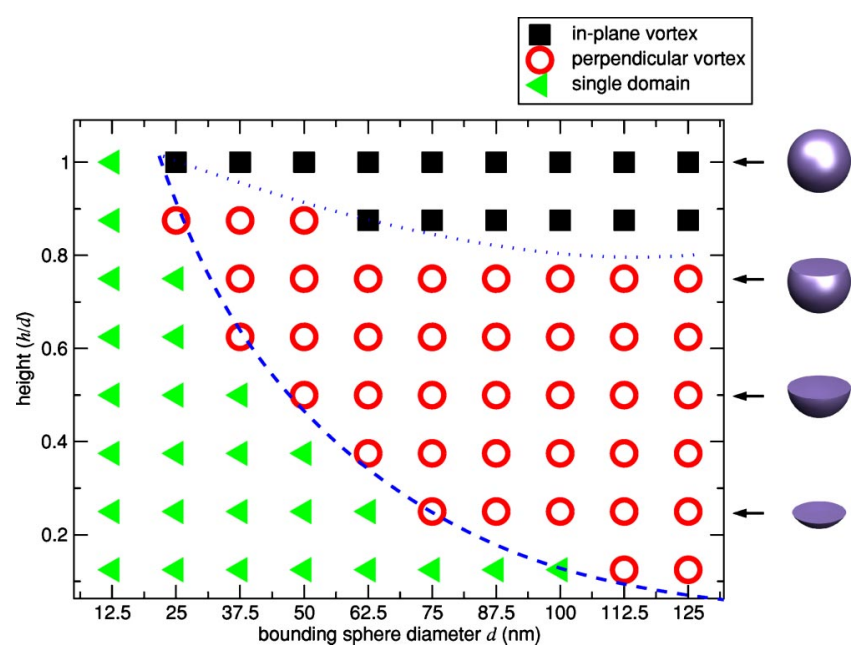

FIG. 1. Phase diagram of remanent states for $\mathrm{Ni}_{50} \mathrm{Fe}_{50}$ permalloy part spheres. The dotted and dashed lines are guides to the eye indicating reversal mechanism boundaries. 


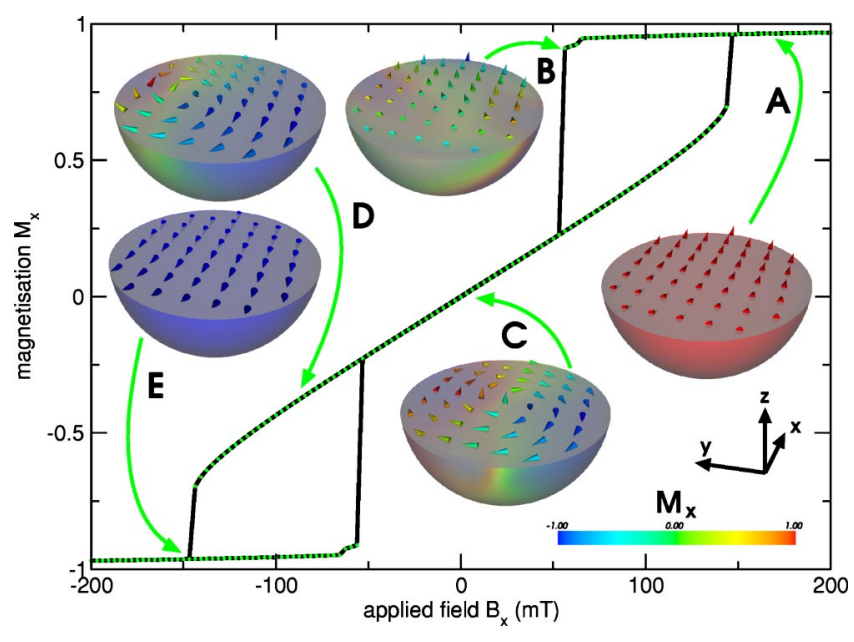

FIG. 2. Reversal mechanism for $d=50 \mathrm{~nm}, h=0.5 d$.

finite difference micromagnetic simulation program OOMMF (Ref. 12) to perform the simulations using the material parameters for $\mathrm{Ni}_{50} \mathrm{Fe}_{50}$ permalloy $\left(J_{s}=1.39 \mathrm{~T}, A=5.85\right.$ $\times 10^{-12} \mathrm{~J} / \mathrm{m}$, and $\left.K_{1}=0 \mathrm{~J} / \mathrm{m}^{3}\right){ }^{14}$ These software packages use the Landau-Lifshitz-Gilbert equation, ${ }^{15}$

$$
\frac{d \mathbf{M}}{d t}=-|\bar{\gamma}| \mathbf{M} \times \mathbf{H}_{\mathrm{eff}}-\frac{|\bar{\gamma}| \alpha}{M_{s}} \mathbf{M} \times\left(\mathbf{M} \times \mathbf{H}_{\mathrm{eff}}\right)
$$

to compute metastable configurations. We perform simulations on part-spherical geometries where the overall diameter $d$ ranges from 12.5 to $100 \mathrm{~nm}$, and the external magnetic field is applied along the "flat" $x$ direction of the part sphere.

Starting from an initially uniform magnetization state pointing in the $+x$ direction, we apply a high magnetic field capable of maintaining a nearly homogeneous magnetization and reduce this field in steps of $1 \mathrm{mT}$ until the magnetization is reversed.

\section{RESULTS}

Figure 1 shows a phase diagram of remanent magnetization states for simulated systems where the height $h$ increases from $1 / 8 d$ to $d$ in $1 / 8 d$ steps and $d$ varies between 12.5 and $125 \mathrm{~nm}$
We observe three distinct reversal mechanisms. Taking $d=50 \mathrm{~nm}$, for $h / d \leqslant 0.375$ the reversal is coherent; all the magnetic moments remain aligned and rotate homogeneously. Between $h / d=0.5$ and $h / d=0.875$ an out-of-plane vortex forms with a core perpendicular to the applied field after an initial energy barrier is overcome and this can freely move around the inside of the part sphere with the applied field. This is similar to the behavior seen in cylindrical particles. ${ }^{4-6,16}$ We will now discuss the reversal mechanism in more detail.

Figure 2 shows the perpendicular vortex reversal behavior. Point $A$ shows the homogeneously aligned state at high applied field, though there is a small C-shaped shift in the $x-z$ direction at the extremeties in order to minimize dipolar energy. At point $B$ the magnetization arranges into an $\mathrm{S}$ shape in the $x-y$ direction, where the magnetic moments at the edges of the half sphere persist in the applied field direction while the moments towards the center are aligned a few degrees away from the $x$ direction into the $y$ direction. Reducing the field further overcomes an energy barrier and a perpendicular (i.e., the core of the vortex points in the $z$ direction) vortex is formed. Point $C$ shows the remanent state of the half sphere with this vortex in the center; the net magnetization in the $x$ direction is now zero. Point $D$ shows the effects of a continued field reduction; the vortex has shifted further into the $y$ direction appropriate for allowing the majority of the magnetic moments to point in the negative $x$ direction. Finally, at point $E$ the magnitude of the negative external field is sufficiently high to remove the vortex from the system and a homogeneously aligned state remains.

Figure 4 (Fig. 3) shows the reversal mechanism with an in-plane vortex for a sphere (i.e., $h / d=1.0$ ). Point $A$ shows a homogeneous alignment of the magnetic moments in the $x$ direction, which persists until point $B$, where the field has been lowered enough to overcome the energy barrier and allow an in-plane (i.e., where the core points in the $x$ direction) vortex to form; this also allows the majority of the magnetization to continue pointing in the $x$ direction. As the field is further reduced, the $x$ component of the magnetiza-

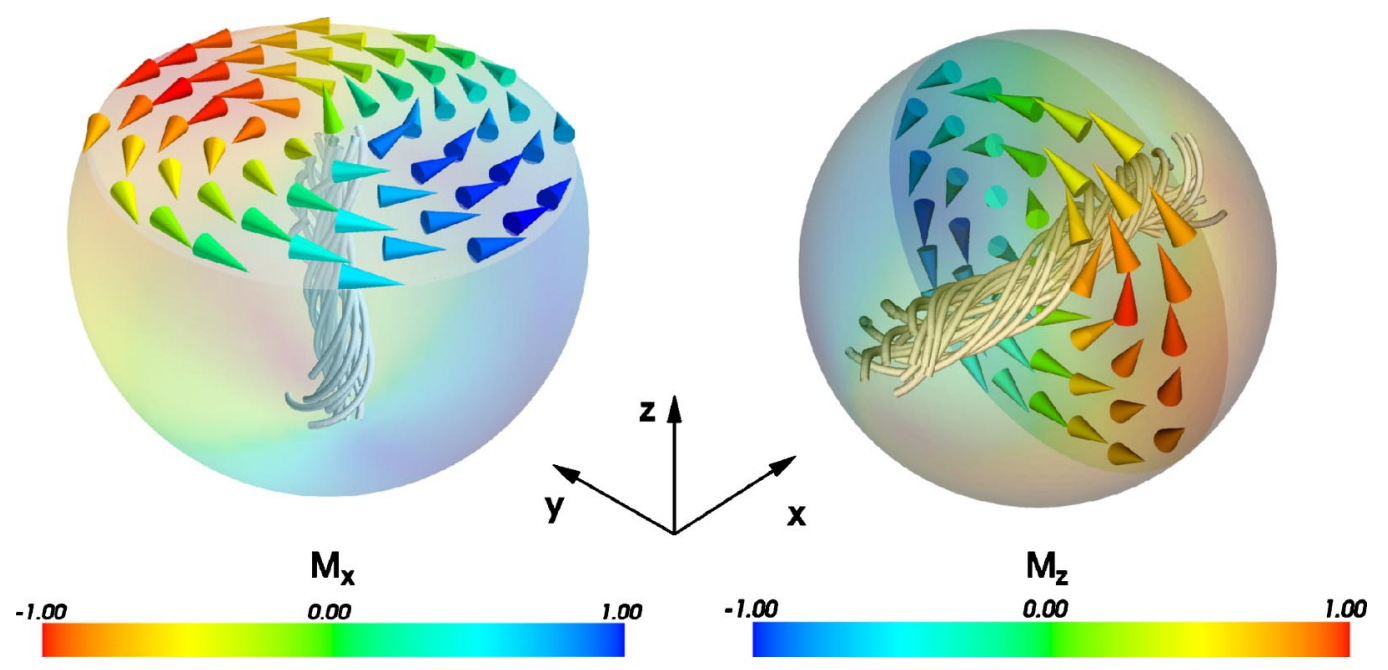

FIG. 3. Remanent magnetization pattern for $d=75 \mathrm{~nm}$ in (left) a $h=3 / 4 d$ part sphere and (right) a full sphere where the initial magnetic field was applied in the $x$ direction. The vortex cores are highlighted by streamlines. 


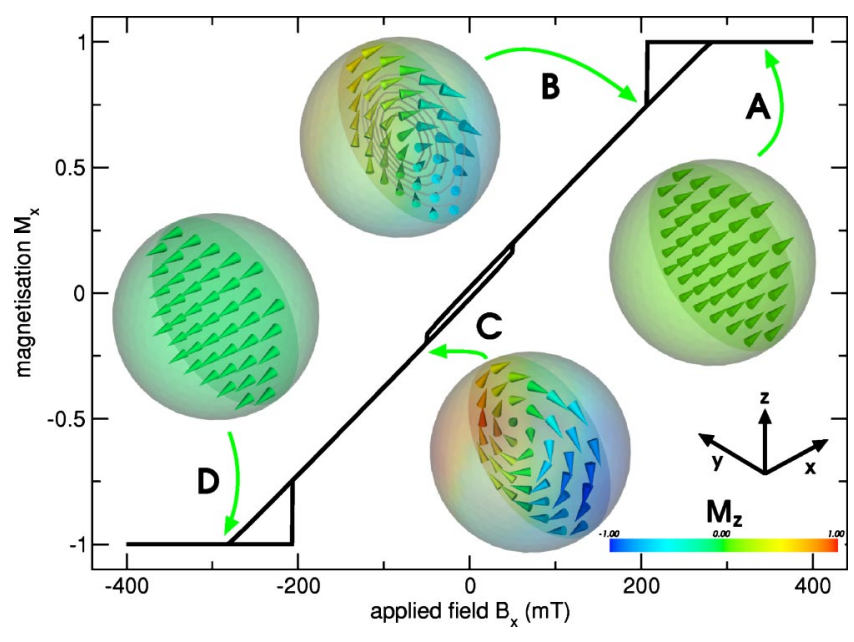

FIG. 4. Reversal mechanism for $d=100 \mathrm{~nm}, h=d$. The center of the vortex core in inset B and C always remains in the center of the $y-z$ plane (see also Fig. 3, right). Inset B shows the isolines of $M_{x}$ in the $y-z$ cutplane to demonstrate this.

tion outside the vortex core continues to follow the applied field; however, the core remains pointing wholly in the direction of the initial applied field. At point $C$, after the field is reduced below zero the core of the vortex flips over, responsible for the "minor" hysteresis loop around $B_{x}=0$. The vortex can exit the system when the magnitude of the negative field is sufficiently high and the magnetization becomes homogeneous (point $D$ ).

Our simulation results agree with the computation of the critical radius ${ }^{17}$ of single-domain to vortex state transition for $\mathrm{Ni}_{50} \mathrm{Fe}_{50}$ in spheres of radius $12.4 \mathrm{~nm}(d=24.8 \mathrm{~nm})$; a singledomain remanent state is observed in our simulations of spheres of diameter $24 \mathrm{~nm}$ and below where the exchange energy is dominant, while an in-plane vortex is in the remanent state when the diameter is $25 \mathrm{~nm}$ as the dipolar energy becomes preponderant.

Figure 5 shows the results computed during the simulation of a half sphere of diameter $50 \mathrm{~nm}$ using two different simulation packages that employ the finite difference method $^{12}$ and the hybrid finite element/boundary element method, ${ }^{13}$ respectively. There is good agreement between the two packages.

\section{SUMMARY}

We have simulated the magnetization reversal in partspherical particles, and three separate remanent statessingle domain, out-of-plane vortex, and in-plane vortexhave been observed.

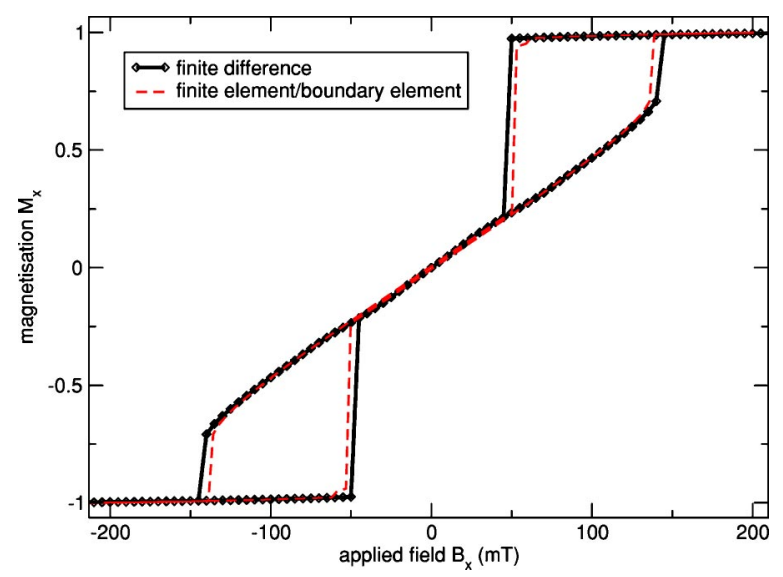

FIG. 5. Hysteresis loops for a $d=50 \mathrm{~nm}$ half sphere obtained with (solid line) the finite difference method and (dashed line) the hybrid finite element/ boundary element method.

As the diameter of the part-spherical particle decreases, a larger $h / d$ is necessary for a vortex to form. As $h$ is increased, the magnetization is more likely to form a vortex. Below a critical radius of $12.4 \mathrm{~nm}$ for $\mathrm{Ni}_{50} \mathrm{Fe}_{50}$, all $h / d$ values will result in a single-domain remanent state.

We have used two different simulation packages and observed good agreement.

${ }^{1}$ A. A. Zhukov, A. V. Goncharov, P. A. J. de Groot, P. N. Bartlett, and M. A. Ghanem, J. Appl. Phys. 93, 7322 (2003).

${ }^{2}$ M. A. Ghanem, P. N. Bartlett, P. A. J. de Groot, and A. Zhukov, Electrochem. Commun. 6, 447 (2004).

${ }^{3}$ R. P. Cowburn, A. O. Adeyeye, and M. E. Welland, New J. Phys. 1, 16 (1999).

${ }^{4}$ R. P. Cowburn, D. K. Koltsov, A. O. Adeyeye, M. E. Welland, and D. M. Tricker, Phys. Rev. Lett. 83, 1042 (1999).

${ }^{5}$ S. P. Li, M. Natali, A. Lebib, A. Péin, Y. Chen, and Y. B. Xu, J. Magn. Magn. Mater. 241, 447 (2002).

${ }^{6}$ K. Ha, R. Hertel, and J. Kirschner, Phys. Rev. B 67, 064418 (2003).

${ }^{7}$ J. C. H. Stapper, J. Appl. Phys. 40, 798 (1969).

${ }^{8}$ I. Eisenstein and A. Aharoni, J. Appl. Phys. 47, 321 (1975).

${ }^{9}$ J. Lam, J. Appl. Phys. 72, 5792 (1992).

${ }^{10}$ R. P. Cowburn, J. Phys. D 33, R1 (2000).

${ }^{11}$ R. P. Cowburn and M. E. Welland, Phys. Rev. B 58, 9217 (1998).

${ }^{12}$ M. J. Donahue and D. G. Porter, OOMMF User's Guide (National Institute of Standards and Technology, Gaithersburg, MD, 1999), interagency Report NISTIR 6376.

${ }^{13}$ W. Scholz, J. Fidler, T. Schrefl, D. Süß, R. Dittrich, H. Forster, and V. Tsiantos, Compos. Sci. Technol. 28, 366 (2003).

${ }^{14}$ R. Skomski and J. M. D. Coey, Permanent Magnetism, Series in Condensed Matter Physics (IOP, Bristol, 1999).

${ }^{15}$ L. D. Landau and E. M. Lifshitz, Phys. Z. Sowjetunion 8, 153 (1935).

${ }^{16}$ R. P. Boardman, H. Fangohr, A. V. Goncharov, A. A. Zhukov, P. A. J. de Groot, and S. J. Cox, J. Appl. Phys. 95, 7037 (2004).

${ }^{17}$ R. C. O'Handley, Modern Magnetic Materials: Principles and Applications (Wiley, New York, 1999), p. 305. 